\title{
Effect of Mixing Solvents on the Structure of Trehalose Tri- isostearate Nonionic Surfactant Reverse Micelles
}

\author{
Rekha Goswami Shrestha, ${ }^{1}$ and Lok Kumar Shrestha ${ }^{2 *}$ \\ ${ }^{I}$ Department of Pure and Applied Chemistry, Faculty of Science and Technology, Tokyo University of \\ Science, 2641 Yamazaki, Noda, Chiba 278-8510, Japan; ${ }^{2}$ International Center for Young Scientists \\ (ICYS), WPI Center for Materials Nanoarchitectonics (MANA), National Institute for Materials Science \\ (NIMS), 1-1 Namiki,Tsukuba Ibaraki 305-0044, Japan; Email: SHRESTHA.Lokkumar@nims.go.jp
}

\begin{abstract}
Effects of mixing fraction of n-decane (Dec) with n-tetradecane (TD) on the shape, size, and internal structure of trehalose tri-isostearate (designated as TQ-3) nonionic surfactant reverse micelles have been investigated using small-angle X-ray scattering (SAXS) technique. TQ-3 self-assembles into a variety of reverse micellar structures in different organic solvents under ambient conditions It was found that a subtle difference in solvent polarity plays a crucial on the structure of reverse micelles. To better understand the solvent dependence of reverse micellar structure, an attempt has been made studying the effects of mixing solvents (alkanes). Three alkanes with different alkyl chain lengths; $C_{10}, C_{12}$, and $C_{14}$, were considered and $C_{10}$ and $C_{14}$ were mixed in different mixing fractions (wt ratio). SAXS measurements were carried out on 5 wt\% TQ-3 systems under ambient conditions. Micellar size and the internal structure (crosssection diameter) were found to depend on mixing fractions of solvents and could be explained in terms of packing parameter.
\end{abstract}

Keywords: Trehalose tri-isostearate, nonionic surfactant, self-assembly, decane, dodecane, tetradecane, small-angle $X$-ray scattering $(S A X S)$, reverse micelles.

\section{Introduction}

Reverse micelles have an opposite structure compared to conventional normal micelles in aqueous systems; comprising a hydrophilic polar core and a lipophilic nonpolar shell. ${ }^{1}$ Studies on reverse micelle formation, structural characterization, and dynamic properties have attracted significant interests in recent years due to their wide ranges of practical applications. Reverse micelles have been used as templates for the tailored synthesis of nanomaterials and also as size controlling microreactors for various aqueous chemical reactions..$^{2-4}$ Investigations have shown that the structures of nanomaterials are closely linked to the structures of template micelles demonstrating the importance of studies of free structure control and tunable parameters for the structural variation of reverse micelles in real practice. Moreover, reverse micelles are good candidates for the encapsulation of polar components or water soluble drugs and hence, finds extensive applications in pharmaceutical product formulations and industries.

\section{* Corresponding author}


Enough evidences on reverse micelles formation are available in the literature; however, most of them deal with studies in ternary mixtures of ionic surfactant/water/oil usually in oil-rich regions, in which water is regarded as an essential component. For examples, ternary mixtures of water/sodium bis(2-ethylhexyl) sulfosuccinate (AOT)/oil or water/lecithin/oil are the mostly considered systems for the studies of reverse micelles or water in oil (W/O) microemulsions so far. ${ }^{5-10}$ However, structure and properties of reverse micelles in nonionic surfactant/oil systems are sparsely studied. Note that hydrophilic part of ethylene oxide (EO)-based conventional nonionic surfactants tends to dissolve in organic solvents and, hence, unable to form any selfassembled structures (reverse micelles) unless a trace amount of water is incorporated into the system. Water hydrates the hydrophilic head-groups drives the self-assembly in nonaqueous media due to dipole-dipole interactions. In such systems, it is the mole ratio of surfactant and water that determines the geometries of reverse micelles and also the dynamic and rheological properties. ${ }^{11}$ Contrary to this, glycerol or sucrose-based nonionic surfactants are solvophobic and self-assemble into various structures in nonaqueous media without water addition from outside. ${ }^{12-14}$ These nonionic surfactants are derived from natural sources and are biodegradable and biocompatible and, hence, have attracted considerable attention from cosmetic and food industries. In the recent study, it has found that trehalose poly-isostearates self-assemble into a variety of reverse micellar structure in different organic solvents under ambient conditions and a subtle difference in solvent polarity plays a crucial role on the structure of reverse micelles. ${ }^{15}$ To better understand the effects of solvent properties, in particular, mixing solvents on the reverse micellar structure, an attempt has been made investigating the shape, size, and internal structure of trehalose tri-isostearate (TQ-3) micelles in mixed solvent systems. Three alkanes with different chain lengths; $\mathrm{C}_{10}, \mathrm{C}_{12}$, and $\mathrm{C}_{14}$ have been considered in this study and $\mathrm{C}_{10}$ and $\mathrm{C}_{14}$ were mixed in different mixing ratio (wt $\%$ ) and SAXS measurements were carried out on 5\% TQ$3 /$ mixed solvent systems under ambient conditions. For the sake of comparison data of TQ-3 in decane, dodecane, and tetradecane are adopted from the literature.

\section{Experimental Methods}

\section{Materials}

Trehalose-tri-isostearate a nonionic surfactant with purity > 99\%, hereafter designated as TQ-3, where 3 represents the number of isostearate chain per surfactant molecule, was a generous gift from the Nisshin OilliO Group, Ltd., Yokohama, Japan. The surfactant was used without further purification. The nonpolar organic solvents, $n$-decane (Dec), $n$-dodecane (DoDec), and $n$ tetradecane (TD), were purchased from Tokyo Chemical Industry, Tokyo, Japan. These oils were $99.5 \%$ pure and used as received. The schematic molecular structure of TQ-3 is given in Scheme 1.

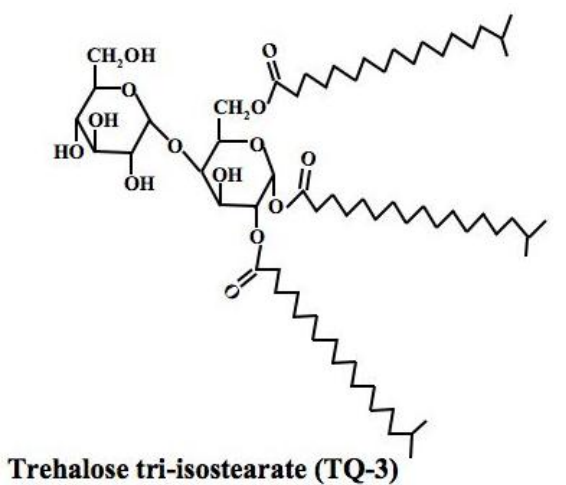

Scheme 1: Schematic molecular structures of trehalose tri-isostearate (TQ-3) 
Sample preparation and identification of equilibrium phase

Organic solvents $n$-decane (Dec) and $n$-tetradecane (TD) were mixed in different mixing fractions (Dec:TD = 20:80, 40:60, 50:50, 60:40, 80:20 (wt ratio)). 5 wt\% TQ-3 solutions were prepared in these solvent mixtures and taken in clean and dry glass ampoules with screw cap. The samples were mixed properly by using dry thermo-bath, vortex mixer and repeated centrifugation to achieve homogeneity. All the samples were placed in a temperature-controlled water bath at $25^{\circ} \mathrm{C}$ for $2 \mathrm{~h}$ before observing the equilibrium phases and SAXS measurements.

\section{SAXS measurements}

SAXS measurements were performed on a SAXSess camera (Anton Paar, Austria) attached to a PW3830 laboratory X-ray generator with a long fine focus sealed glass X-ray tube $\left(K_{\alpha}\right.$ wavelength of $0.1542 \mathrm{~nm}$ ) and operated at $40 \mathrm{kV}$ and $50 \mathrm{~mA}$. An equipped Göbel mirror and a block collimator enabled us to obtain a focused monochromatic X-ray beam of $\mathrm{Cu}-K_{\alpha}$ radiation $(\lambda=0.1542 \mathrm{~nm})$ with a well-defined line-shape. A thermostated sample holder unit was used to control the sample temperature. The 2-D scattering pattern was recorded on an imaging-plate and integrated into to 1-D scattered intensities $I(q)$ as a function of the magnitude of the scattering vector $q=(4 \pi / \lambda) \sin (\theta / 2)$ using SAXSQuant software (Anton Paar), where $\theta$ is the total scattering angle. All measured intensities were semi-automatically calibrated for transmission by normalizing a zero- $q$ attenuated primary intensity to unity, by taking advantage of a semi-transparent beam stop. All $I(q)$ data were corrected for the background scattering from the capillary and the solvents. Furthermore, the absolute scale calibration was made using water as a secondary standard. Real-space structural information so called pair-distance distribution functions, $p(r)$, were obtained by generalized indirect Fourier transformation (GIFT) evaluation of the SAXS data. ${ }^{16,17}$ This procedure relies on a basic equation of one-component globular particle systems, $I(q)=n P(q) S(q)$, and its extension to polydisperse systems, where $P(q)$ is the averaged form factor, $S(q)$ is the averaged static structure factor, and $n$ is the number of particles in unit volume. To suppress the influence of interparticle interference scattering on the evaluation of $p(r)$ that generally leads to oscillations and to a highly underestimated maximum size of the scattering object, an interaction potential model for $S(q)$ was to be considered in the analysis for which an averaged structure factor of hard-sphere interaction potential model, ${ }^{18,19}$ and Percus-Yevick closure relations to solve Ornstein-Zernike equation were used. The detailed theoretical description on the method is described elsewhere. ${ }^{20,21}$

\section{Results and Discussion}

Isothermal phases in the dilute regions

In order to confirm whether the proposed systems exist in isotropic single phase solution, equilibrium phases of $5 \mathrm{wt} \%$ TQ-3 in mixture of Dec and TD at different mixing ratios; Dec:TD $=20: 80,40: 60,50: 50,60: 40,80: 20$, were identified by visual inspection observing samples through a crossed polarizer at $25{ }^{\circ} \mathrm{C}$. It has found that $5 \mathrm{wt} \%$ TQ-3 in aforementioned solvent mixtures form isotropic solutions of reverse micelles. 


\section{Reverse micellar structure}

Figure 1 shows the normalized X-ray scattered intensities, $I(q) \mathrm{s}$, and the real space pairdistance distribution functions, $p(r) s$, obtained from the GIFT evaluation of the SAXS data for 5 wt\% TQ-3 in mixed Dec and TD systems at different mixing ratios (Dec:TD = 20:80, 40:60, $50: 50,60: 40,80: 20$ (wt ratio) ) at $25^{\circ} \mathrm{C}$. For the sake of comparison data for $5 \mathrm{wt} \%$ TQ-3 in Dec, DoDec, and TD are adopted from Ref. 15. Note that strong $q$ dependence of $I(q)$ in the scattering curves is a clear signature of micellar aggregates; in the absence of aggregates structure, $I(q)$ is independent of $q$ in the SAXS regime. Absence of peaks in the low- $q$ regime of the $I(q)$ curves demonstrate a negligible inter-micellar interactions though the SAXS measurements were carried out in semi-dilute concentrations (5\% surfactant). As it can be seen in Figure 1a-b, scattering behavior differs depending on the mixing fraction of Dec with TD. In the absence of Dec, the low- $q$ slope of $5 \mathrm{wt} \%$ TQ-3/TD system is close to $\sim q^{-1}$ indicating elongated geometry of the micelles. Similarly, in $5 \mathrm{wt} \%$ TQ-3/Dec system, the low- $q$ slope is $>$ $q^{-0}$ showing that micelles are still elongated geometry ellipsoidal prolate. ${ }^{15}$ When Dec and TD are mixed in different mixing ratios as mentioned earlier, the low- $q$ slope lies in between $q^{-0}$ and $q^{-1}$ at all mixing fractions. This shows that at all mixing fractions, micelles exist in ellipsoidal prolate geometry, which is further supported by form factor curves $P(q)$; all the curves follow the similar behavior, i.e., micellar shape remains apparently the same despite a wide variation in the solvents mixing ratios, the only aspect ratio and number density of micelles vary. Nevertheless, since the scattering intensities in the high- $q$ cross section regime $1.5 \leq q \leq 3.0 \mathrm{~nm}^{-}$ ${ }^{1}$ shift towards lower- $q$ region (forward direction) with increasing Dec:TD ratio, the internal cross section structure of micelles seems to increase.

Shape, size, and internal structure of TQ-3-based reverse micelles depending on mixing ratios of Dec with TD can best be seen in real space $p(r)$ functions shown in Figure 1c-d. The $p(r)$ curves exhibit typical feature of elongated ellipsoid prolate type micelles judged from pronounced peak in the low- $r$ side with an extended downward convex tail to the higher- $r$ side. $^{22,23}$ In case spherical geometry, $p(r)$ curve exhibits symmetric bell shape. On the other hand, pronounced peak in the low- $r$ side with an extended linear tail in the higher- $r$ region of the $p(r)$ curve is the typical of cylindrical micelles. ${ }^{15}$ Minute observations of $p(r)$ curves reveal that reverse micelles of $5 \mathrm{wt} \%$ TQ-3 in TD, DoDec, and Dec exhibit ellipsoid prolate geometry with maximum dimensions, $D_{\max }$, ca. 7.75, 6.79, and $5.82 \mathrm{~nm}$, respectively (see inset of Fig. 1c), whereas cross section diameter ca. $2.6 \mathrm{~nm}$ is estimated from the inflection point seen after the maximum of $p(r)$ curve. ${ }^{15}$ When TD is replaced with mixture of Dec:TD $=20: 80$, aspect ratio of prolate ellipsoid micelles decreases as $D_{\max }$ is estimated ca. $7.21 \mathrm{~nm}$. With further increase in Dec content in the system, $D_{\max }$ continue to decrease and attains exactly the same value of DoDec system at Dec:TD $=50: 50$ system. This shows that Dec:TD $=50: 50$ system mimics the DoDec system as far as micellar size is concerned. However, situation turns out to be fairly different in terms of cross section structure. The inflection points seen after the maximum of the $p(r)$ curves as indicated by broken lines in Fig. 1c, which semi-quantitatively measures the cross section diameter of micelles tends to increase in parallel to the mixing fraction of Dec, attains maximum value at Dec:TD $=60: 40$, and decrease. This behavior can clearly be seen in normalized $p(r)$ curves $\left(p(r) / p\left(r_{\max }\right)\right)$ presented in Fig. 1d. Note that position of maximum of $p(r)$, i.e., $r_{\max }$ changes with mixing fractions of Dec or TD see inset of Fig. 1d. 

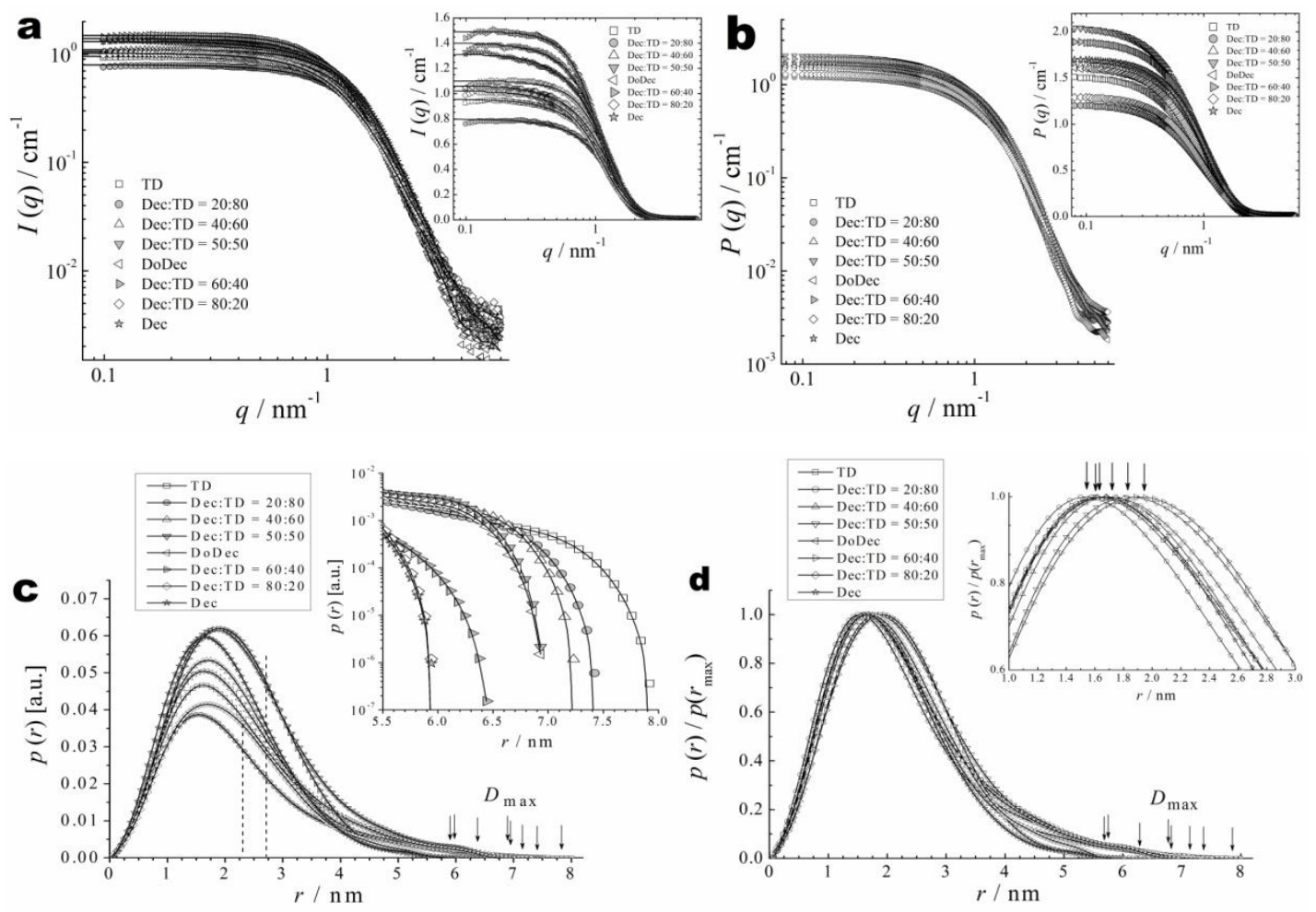

Figure 1: Effects of mixing solvents on the reverse micellar structure: (a) normalized X-ray scattered intensities, $I(q)$, (b) the calculated form factor $P(q)$ for $n$ particles existing in unit volume $n P(q)$, (c) the corresponding $p(r)$-functions, and $(d)$ the normalized $p(r)$ functions, $p(r) / p\left(r_{\max }\right)$ for 5 wt\% TQ-3 in mixed Dec and TD systems at different mixing ratios (Dec:TD = 20:80, 40:60, 50:50, 60:40, 80:20) at $25^{\circ} \mathrm{C}$. The solid lines in panel (a) represent the GIFT fit. The broken lines and arrows in panel (b) highlight the maximum dimensions, $D_{\max }$, and maximum cross-sectional diameters, $D_{\mathrm{cmax}}$, of the micellar core. Insets of panels $(a)$ and $(b)$ demonstrate the low-q behavior. Inset of panel $(c)$ shows $p(r)$ in the higher-r regime, which makes easier to estimate $D_{\max }$, and inset of panel $(d)$ shows normalized $p(r)$ curves in the lower-r regime. The data for Dec, DoDec, and TD are adopted from Ref. 15.

The observed micellar shrinkage with increase in mixing fractions of Dec with TD can be explained in terms of modification in critical packing parameter $\left(c p p c p p=v / a_{\mathrm{s}} l_{\mathrm{c}}\right.$ where $v$ is the volume of the lipophilic portion of the amphiphile, $a_{\mathrm{s}}$ and $l_{\mathrm{c}}$ are the area of hydrophilic headgroup and the extended chain length of the lipophilic portion, respectively) or micellar curvature. ${ }^{24}$ The penetration phenomenon to the lipophilic chain of surfactant molecules modifies the cpp, and generally makes spontaneous curvature negative. When Dec is mixed with TD, the average alkyl chain length of oil changes as a result mixed oils have different penetration tendency into the hydrophilic/lipophilic interface of micelles so as to avoid energetically unfavorable direct contact between oils and hydrophilic group of the surfactant and modifies the micellar geometry. In the studies of solvent dependence of reverse micellar structure, it has found that polar oils or short chain hydrocarbon oils can penetrate to the 
lipophilic chain of surfactant thereby increasing cpp form globular micelles, i.e., micellar curvature becomes more negative. Here TD due to its long carbon chain $\left(\mathrm{C}_{14}\right)$ cannot penetrate to the lipophilic chain of TQ-3 surfactant and, hence, self-assembles into elongated ellipsoidal prolate type of reverse micelles with high aspect ratio. Note that the average alkyl chain length of oil tends to decrease upon incorporation of small fraction of Dec $\left(\mathrm{C}_{10}\right)$ in TD and continue to decreases with the mixing fractions of Dec in total system. As a result, the penetration tendency increases and cpp increases. Under this condition micellar curvature becomes more curved and micellar size tends to decrease. At Dec:TD $=50: 50$, the average carbon chain of oil mixture is equivalent of that of DoDec $\left(\mathrm{C}_{12}\right)$ and, hence, micellar size of these systems are essentially the same.

\section{Direct cross section analysis}

Cross section core diameters of TQ-3-based reverse micelles in pure solvents (Dec, DoDec, and TD) and mixtures of Dec and TD are estimated ca. $2.2-2.8 \mathrm{~nm}$ from the inflection points after the maximum of total $p(r)$ curves (Fig. 1c-d). For quantitative estimation of the cross section structure of the micellar core, indirect Fourier transformation (IFT) method was used. The cross-sectional pair-distance distribution function, $p_{\mathrm{c}}(r)$, can directly be calculated from the experimental scattered intensity $I(q)$ using this method. ${ }^{15}$ The further deconvolution procedure of the $p_{\mathrm{c}}(r)$ yields the radial difference electron density distribution profile, $\Delta \rho_{\mathrm{c}}(r){ }^{25,26}$ Figure 2 shows the resulting $p_{\mathrm{c}}(r)$ and $\Delta \rho_{c}(r)$ for $5 \mathrm{wt} \%$ TQ-3 in the mixture of Dec and TD at different mixing ratios (Dec:TD $=20: 80,40: 60,50: 50,60: 40,80: 20)$ at $25^{\circ} \mathrm{C}$. Here again for the sake of comparison data for $5 \mathrm{wt} \%$ TQ-3 in DoDec, and TD are adopted from Ref. 15 systems.
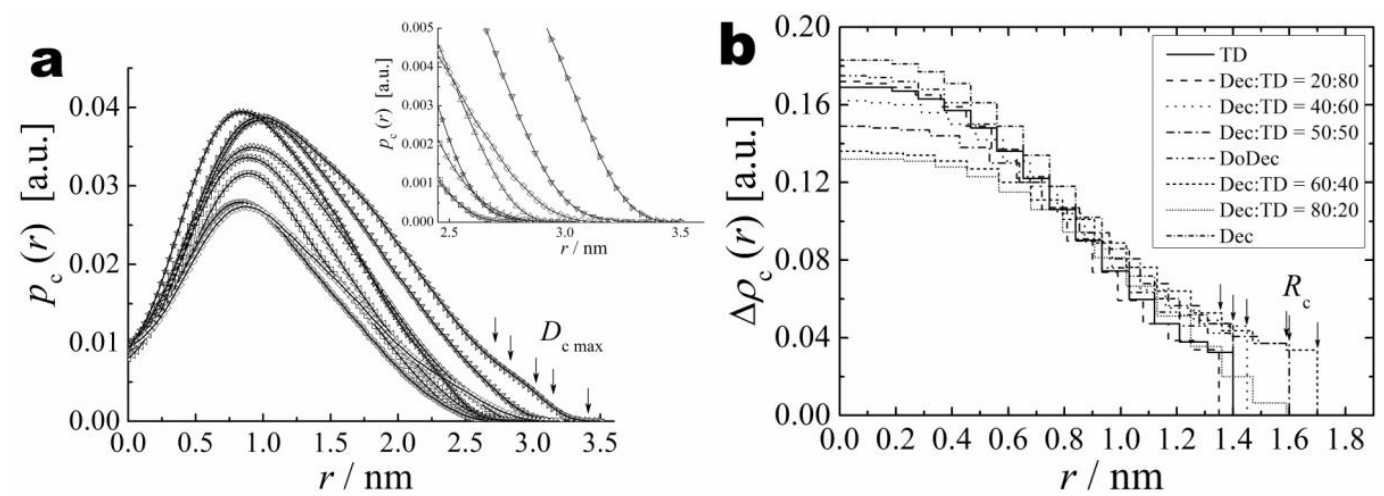

Figure 2: (a) The cross-sectional pair-distance distribution function, $p_{c}(r)$, of $5 w t \%$ TQ-3 the mixture of Dec and TD at different mixing ratios (Dec:TD = 20:80, 40:60, 50:50, 60:40, 80:20) at $25{ }^{\circ} \mathrm{C}$, and $(b)$ the resulting cross-section radial electron density profile, $\square \square c(r)$, calculated with the deconvolution procedure of $p_{c}(r)$ using program DECON. Symbols of panel $(a)$ correspond to the systems mentioned in Figure 1. Downward arrows in panels (a) and (b) represent the maximum core diameter, $D_{\text {cmax }}$, and core radius, $R_{c}$, respectively. The data for DoDec, and TD are adopted from Ref. 15.

The $p_{\mathrm{c}}(r)$ functions represent typical homogeneous aggregates with variable maximum core diameter depending on the mixing fraction of solvents. From the position of $D_{\text {cmax }}$ in the higher- 
$r$ regime of the $p_{\mathrm{c}}(r)$ curves values of cross section diameters in TD, DoDec, and Dec are estimated ca. $2.60 \mathrm{~nm}$, which are essentially the same with those roughly estimated from the inflection point of total $p(r)$ curves. Cross section diameter of Dec:TD $=20: 80$ is comparable to that of pure solvent systems; however, with further increase in Dec fraction into the system, $D_{\text {cmax }}$ increases and attains a maximum value ca. $3.39 \mathrm{~nm}$ in Dec:TD $=60: 40$ system and decreases.

Note that the extended length of trehalose group is estimated to be ca. $1.3 \mathrm{~nm}$. The micellar core cross section diameter observed is higher than the twice of extended length of trehalose group and cannot be explained unless presence of water (present as impurity) in the surfactant and oils or polydispersity in core cross section diameters are taken into account. The positive values of $\Delta \rho_{c}(r)$ in the entire $r$-range as shown in Figure $2 \mathrm{~b}$ confirm the electron rich hydrophilic reverse micellar core. As naturally expected from $p_{\mathrm{c}}(r)$, the maximum reverse micellar core radius, $R_{\mathrm{c}}$, is estimated ca. $1.30 \mathrm{~nm}$ for pure solvent systems, which is exactly half of the $D_{\text {cmax }}$. The $R_{\mathrm{c}}$ in mixed solvent systems followed asimilar trend of variation in $D_{\text {cmax }}$.

\section{Conclusion}

Effects of mixing solvents on the shape, size, and internal structure of trehalose triisostearate (TQ-3)-based nonionic surfactant reverse micelles have been investigated using small-angle X-ray scattering (SAXS) technique. Real space structural information of the micellar aggregates has been obtained by inverse Fourier transformation method. It has found that reverse micellar geometry largely depend on solvent properties. A subtle variation in alkyl chain length of oil modifies the reverse micellar geometry noticeably. TQ-3 self-assembled into elongated ellipsoidal prolate geometry in alkanes and the aspect ratio of elongated structure tends to increase with increase in average alkyl chain length of oil. This paper has demonstrated that tuning mixing fraction of alkanes one can freely control the structure of reverse micellar aggregates and the modulation in the structure of micelles could be explained in terms of critical packing parameter.

\section{Acknowledgements}

Authors are thankful to The Nisshin OilliO Group, Co. Ltd., Yokohama for the supply of surfactant. LKS thanks International Center for Young Scientists (ICYS), National Institute for Materials Science (NIMS) and International Center for Materials Nanoarchitectonics (MANA) for financial support. Fruitful discussions with Prof. Kenji Aramaki, Yokohama National University, and Dr. Takaaki Sato, Shinshu University are highly acknowledged.

\section{References}

1. J. N. Israelachvili, D. J. Mitchell, B. W. Ninham, J. Chem. Soc. Faraday Trans.2, 1976, 72, 1525.

2. P. L. Luisi, B. E. Strab (Eds.) Reverse Micelles: Biological and Technological relevance of Amphiphilc Structures in Apolar Media; Plenum Press: New York, 1987. 
3. M. A. López-Quintela, C. Tojo, M. C. Blanco, L. García Rio, J. R. Leis, Current Opinion in Colloid and Interface Science, 2004, 9, 264.

4. I. Lisiecki, M. P. Pileni, J. Am. Chem. Soc.,1993, 115, 3887.

5. R. E. Riter, J. R. Kimmel, E. P. Undiks, N. E. Levinger, J. Phys. Chem. B., 1997, 101, 8292.

6. M. Kanamaru, Y. Einaga, Polymer, 2002, 43, 3925.

7. J. P. Cason, C. B. Roberts, J. Phys. Chem. B., 2000, 104, 1217.

8. Q. Li, T. Li, J. Wu, J. Phys. Chem. B., 2000, 104, 9011.

9. T. De, A. Maitra, Adv. Colloid Interface Sci., 1995, 59, 95.

10. S-H. Tung, Y-E. Huang, S. R. Raghavan, J. Am. Chem. Soc., 2006, 128, 5751.

11. R. Scartazzini, P. L. Luisi, J. Phys. Chem., 1988, 92, 829.

12. L. K. Shrestha, J. Nepal Chem. Soc., 2008/2009, 23, 74.

13. K. Hashizaki, T. Chiba, H. Taguchi, Y. Saito, Colloid Polym. Sci., 2009, 287, 927.

14. K. Hashizaki, H. Taguchi, Y. Saito, Colloid Polym. Sci., 2009, 287, 1099.

15. L. K. Shrestha, T. Sato, M. Dulle, O. Glatter, K. Aramaki, J. Phys. Chem. B, 2010, 114, 12008.

16. G. Fritz, A. Bergmann, O. Glatter, J. Chem. Phys. 2000, 113, 9733.

17. O. Glatter, G. Fritz, H. Lindner. P.J. Brunner, R. Mittelbach, R. Strey, S.U. Egelhaaf, Langmuir, 2000, 16, 8692.

18. P. N. Pusey, H. M. Fijnaut, A. Vrij, J. Chem. Phys., 1982, 77, 4270.

19. P. Salgi R. Rajagopolan, Adv. Colloid Interface Sci., 1993, 43, 169.

20. O. Glatter, Progr. Colloid Polym. Sci., 1991, 84, 46.

21. O. Glatter, J. Appl. Cryst., 1981, 14, 101.

22. L. K. Shrestha, T. Sato, K. Aramaki, J. Phys. Chem. B., 2007, 111, 1664.

23. L. K. Shrestha, T. Sato, K. Aramaki, Phys. Chem. Chem. Phys., 2009, 11, 4251.

24. J. Israelachvili, Intermolecular and Surface Forces; Academic Press: San Diego, CA, 1991.

25. O. Glatter, J. Appl. Crystallogr., 1981, 14, 101.

26. O. Glatter, B. Hainisch, J. Appl. Crystallogr., 1984, 17, 435. 